\title{
Demonstration of Dynamic Targeting in an Online Social Medium
}

\section{AUTHors:}

D. J. Higham, P. T. Laflin, F. K. Ainley, A. L. Otley, A. V. Mantzaris

\section{Abstract}

We will demonstrate new software for analysing social media networks, such as Twitter and Facebook, in real time to calculate influence ratings that identify influential individuals and assess sentiment within a conversation. The software utilises the new dynamic centrality measures [1,2] developed at the Universities of Reading and Strathclyde and allows a changing social network to be investigated quickly and accurately. The influence score is different to those produced by Klout and Peer Index. The score calculated by the software focuses on a specific conversation rather than a specific individual. When assessed by social media experts, the scores produced by the software have been verified to be more representative and realistic than Klout and Peer Index in relation to specific conversations.

\section{Concept}

Conference delegates will Tweet using the hashtag \#socinfo2012. In real time, as Tweets are published by delegates, the conversation about the conference can be visualised through a web

browser, for delegates to observe.

Using dynamic centrality calculations, performed on the raw Tweet data, a real time list of influential Tweeters will be produced and updated once every 10 minutes for all to see.

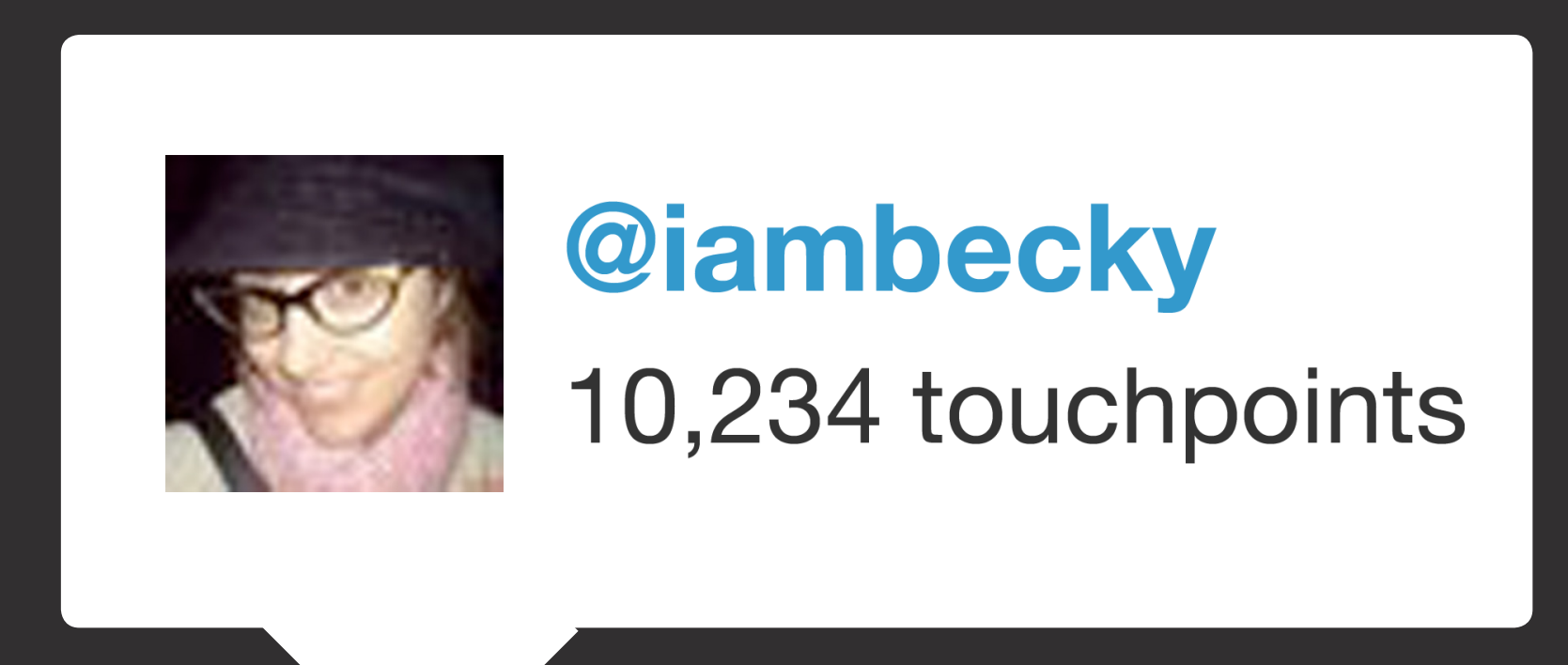

\section{Key facts}

The tool monitors messages sent in social networks for a selected topic. It captures the dynamic nature of the influence users on each other.

Calculating the influence nodes have on each other and their ability to spread infomation is important for assessing the value of targeted advertising campaigns.

Recent developments in centrality measures for dynamic networks has provided a way to measure this influence.

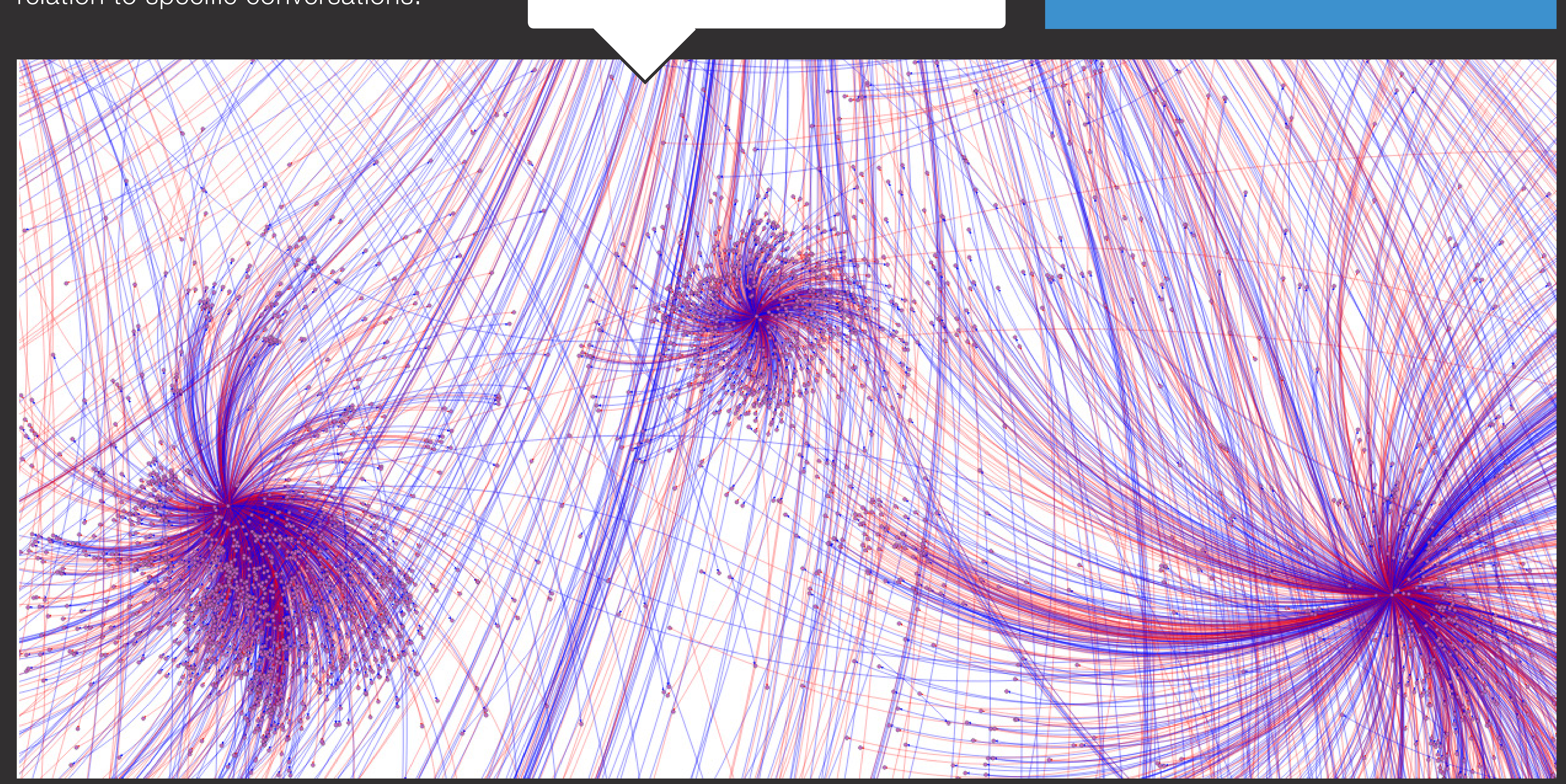

\section{References}

This demo supports the paper "Dynamic Targeting in an Online Social Medium" to be presented as a full paper to this conference on 6 December 2012 in research session 4.

[1] P. Grindrod, M. C. Parsons, D. J. Higham and E. Estrada, Communicability across evolving networks, Phys. Rev. E, 83, 2011 [2] P. Grindrod, and D. J Higham, A matrix iteration for dynamic network summaries, SIAM Review, to appear. [3] L. Katz, A New Status Index Derived from Sociometric Index. Psychometrika, 1953.
VMPOOP

B. $00 \mathrm{~m}$ University of $\begin{aligned} & \text { Universityo of } \\ & \text { Strathchyde } \\ & \text { Glasgow }\end{aligned}$ 